\section{TORRE DE RTVE EN EL POLIGONO 35 DE LA AVENIDA DE LA PAZ MADRID/ESPAÑA}

$504-9$

\section{sinopsis \\ El presente número de la Revista Informes ocupa su sección "Obras y proyectos», con carácter monográfico, a la recientemente terminada torre de RTVE en Madrid. En la misma se contemplan, de una manera exhaustiva, todos los aspectos que implican la construcción de una obra de características tan singulares, como son los de planteamiento general y de concepto, constructivos y estructurales en todas sus vertientes, urbanísticos y plásticos, cada uno de los cuales es desarrollado por los técnicos y especialistas que han intervenido tanto en el proyecto como en la ejecución.}

Se proporciona una definición muy precisa de las caracteristicas geométricas de la torre, aportándose datos completos referidos a la cimentación, fuste, copa o capitel y plataformas, escaleras y núcleo de ascensores, mástil para antenas, etc., con un apunte concreto de las solicitaciones mecánicas que soporta cada elemento de las bases o hipótesis de cálculo, de la organización y medios auxiliares empleados, con especial hincapié en la descripción del sistema de encofrado deslizante utilizado, así como de los aspectos de seguridad y organización de la obra.

\section{ANTECEDENTES}

La torre de RTVE en Madrid surge de la necesidad, recogida en el Plan de Extensión y Renovación de la Red de RTVE, de instalar un Centro Nodal de Radioenlaces en Madrid.

Hasta el momento el servicio se venia prestando con los centros de Prado del Rey y Paseo de La Habana. Las continuas edificaciones en Madrid, cada vez de mayor altura, suponen un obstáculo importante en el perfil Prado del Rey-Paseo de La Habana.

La Dirección Técnica de RTVE estudió diversas soluciones destinadas a elegir un emplazamiento que resolviese de forma definitiva los problemas inherentes a las funciones que ha de desarrollar un Centro Nodal de Radioenlaces de una Red que, como la de RTVE, dispone ya de un elevado número de circuitos y cierta complejidad en la conmutación y tráfico de señales radioeléctricas de programas de Radio y Televisión.

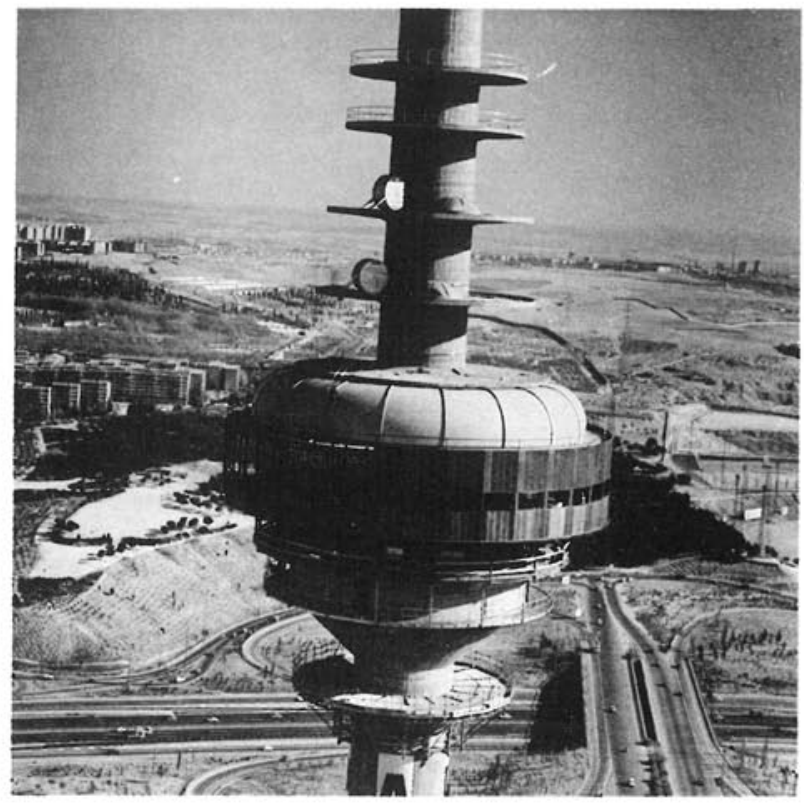

Simultáneamente la Dirección General de RTVE gestionó la compra de un terreno dentro del casco urbano de Madrid que pudiera servir de base para un centro informativo de RTVE en el Campeonato Mundial de Fútbol de 1982, llegándose a la adquisición del Poligono número 35 de la Avenida de la Paz (Madrid).

La Dirección Técnica de la Red se planteó la posibilidad de estudiar la instalación del Centro Nodal de Radioenlaces y Centro Emisor de Madrid en dicho poligono, mediante la construcción de una torre de aproximadamente $200 \mathrm{~m}$ de altura.

Desde el punto de vista de tráfico de señales radioeléctricas la erección de la torre mencionada permite disponer en el Control Central, emplazado en dicho poligono, de todas las señales de los distintos centros regionales simultáneamente, asi como las de la Red de Eurovisión y del Satélite Intel.

Otra ventaja es la disponibilidad de un punto óptimo para la difusión de programas de Radio en frecuencia modulada y de televisión tanto en UHF como en VHF, con una cobertura de casi la totalidad del casco urbano de Madrid y sus alrededores.

También se ha previsto que la torre permita la conexion mediante enlaces móviles, del Control Central con posibles puntos de origen de retransmisiones (partidos de fútbol, conciertos, etc.).

De esta forma se convoco un Concurso de Proyecto y Obras de la torre, tanto de su estructura como de sus instalaciones, cerramientos y acabados necesarios para hacer posible la instalación de equipos y su funciona- 
d) Plataforma de equipos de enlaces de retransmisiones

Se necesita para estos equipos una plataforma cerrada con una cubierta de poliéster reforzado con fibra de vidrio y climatizada, con vuelo cubierto de $9,00 \mathrm{~m}$ y una altura de $4,80 \mathrm{~m}$. Esta planta requiere la instalación de un suelo técnico de $0,40 \mathrm{~m}$ de altura para poder llevar el cableado necesario a los equipos y a los sistemas de refrigeración.

e) Plataforma de equipos transmisores

En esta plataforma se requiere un vuelo de $11,00 \mathrm{~m}$ y debe ser cerrada y climatizada necesitando una altura de $5,20 \mathrm{~m}$ y la instalación de un suelo técnico como en la plataforma anterior.

f) Plataforma de equipos auxiliares

Esta plataforma se necesita para la instalación de los equipos de climatización de las plantas anteriores y de refrigeración de los equipos, así como de baterias, almacén, aseos, etc. Se requiere un vuelo de $8,50 \mathrm{~m}$ y una altura de $3,60 \mathrm{~m}$ y tiene que ir cerrada pero con rejillas de ventilación para los climatizadores.

g) Plataforma de otros servicios

Para una posible ampliación futura se necesita una planta de $5,50 \mathrm{~m}$ de vuelo y $3,60 \mathrm{~m}$ de altura cerrada y teniendo en la parte exterior una pasarela metálica de $2,00 \mathrm{~m}$ de anchura para la posible instalación de antenas y parábolas provisionales.

h) Fuste

En el fuste se necesita una serie de orificios a distintas alturas para poder en un futuro instalar antenas y parábolas para servicios auxiliares de telecomunicación. También se requiere instalar, en el interior, tres estructuras metálicas en forma de escalera vertical de 2,00 $\mathrm{m}$ de anchura, para poder sujetar los diferentes cables de alimentación eléctrica de conexión de señales entre los equipos, las antenas $y$ el edificio situado junto a la torre.

\section{i) Accesos}

Para el acceso a las distintas plataformas de la torre se necesitan un ascensor de $1.000 \mathrm{~kg}$ de carga útil y una escalera en el interior del fuste que permita el acceso en caso de averia del ascensor y la colocación y mantenimiento de los cables en las estructuras verticales.

\section{BASES DE DISEÑO ESTRUCTURAL}

Desde el punto de vista estructural la torre se compone de cinco elementos básicos:
a) Antena
b) Plataformas para parábolas
c) Plataformas para equipos
d) Fuste
e) Cimentación

Describimos las soluciones estructurales adoptadas para cada elemento en la torre de RTVE de Madrid.

\section{Antena}

La antena es una estructura metálica a base de perfiles angulares. El tipo de celosia de las caras adoptado es el más adecuado para recibir los elementos radiantes que soportará la antena.

Esta estructura metálica tiene cuatro tramos diferenciados.

El tramo superior de sección cuadrada, de $0,60 \mathrm{~m}$ de lado y $10 \mathrm{~m}$ de longitud, está formado por cuatro ángulos en sus esquinas empresillados mediante pletinas metálicas soldadas. Este tramo superior se fraccionó en tres partes iguales para facilitar el montaje.

Inmediatamente debajo se dispone un elemento de transición troncopiramidal de $1,50 \mathrm{~m}$ de altura que enlaza este tramo con el intermedio de $15,0 \mathrm{~m}$ de altura $\mathrm{y}$ sección transversal cuadrada de $1,50 \mathrm{~m}$ de lado. A continuación existe un nuevo elemento de transición, también de $1,50 \mathrm{~m}$ de alto, troncopiramidal, con bases cuadradas de $1,15 \mathrm{~m}$ y $2,30 \mathrm{~m}$. El tramo inferior de $13,5 \mathrm{~m}$ de alto y sección transversal cuadrada de $2,30 \mathrm{~m}$ de lado, termina en su parte inferior en un tramo de ensanche de $3,0 \mathrm{~m}$ de altura, constituido por las cuatro patas de apoyo de la estructura. Estando éstas en una circunferencia del mismo diámetro que el de coronación del fuste de hormigón.

Salvo el tramo superior, todos los demás llevan uniones atornilladas, para facilitar el montaje.

Los perfiles laminados de la estructura son de acero de calidad A 42 b y se adoptó una tensión admisible de $1.600 \mathrm{~kg} / \mathrm{cm}^{2}$.

La tornilleria es de calidad 5,6 y se tomó como tensión cortante admisible $1445 \mathrm{~kg} / \mathrm{cm}^{2}$. La presión de los tornillos contra paredes se limitó a la tensión de fluencia de las barras, es decir, $2.600 \mathrm{~kg} / \mathrm{cm}^{2}$. Todos los tornillos Ilevan arandela plana y "grover".

Toda la estructura se ha galvanizado en caliente con un espesor minimo de zinc de 150 micras. Posteriormente a su montaje se dio la mano definitiva de pintura en franjas alternadas rojas y blancas como balizamiento diurno.

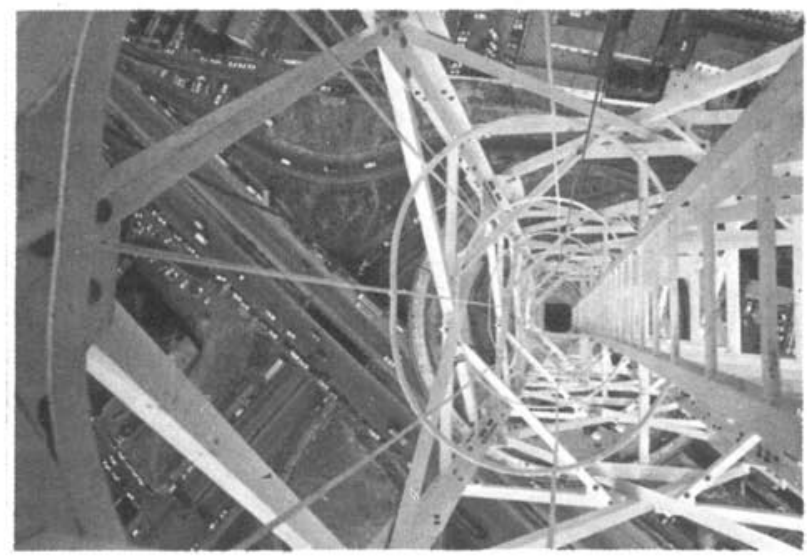


Informes de la Construcción/341

Las hipotesis consideradas han sido:

- Peso propio de la estructura metálica.

- Peso propio de los elementos radiantes que soporta la estructura.

- Acción del viento con una carga dinámica de 150 $\mathrm{kg} / \mathrm{m}^{2}$ sobre estructura y elementos radiantes. Se ha considerado que parte de la estructura está protegida de la acción del viento por las antenas y radomos.

Las cargas totales de viento consideradas han sido:

- Sobre el tramo superior de 0,60 m de lado: $180 \mathrm{~kg} / \mathrm{m}$

- Sobre el tramo intermedio de 1,15 m de lado: $288 \mathrm{~kg} / \mathrm{m}$

- Sobre el tramo inferior de 2,30 m de lado: $482 \mathrm{~kg} / \mathrm{m}$

La obtención de esfuerzos en las barras se ha realizado con auxilio de un ordenador.

Los esfuerzos más desfavorables para los montajes, los correspondientes al viento diagonal, añadiendo la parte correspondiente del peso de la barra para las cargas de compresión y restando éste para las de tracción.

Para las diagonales y péndolas horizontales es más desfavorable el viento perpendicular a una cara.

El cálculo de las barras sometidas a compresión se realizo por el método $w$.

Las barras sometidas a tracción se han calculado considerando la sección neta, que es la total de la barra menos la sección perdida por los taladros.

Finalmente se comprobo que la deformación angular máxima de la estructura, sumada a la del fuste de hormigón de la torre, no superaba el valor dado como admisible de 0,3 grados sexagesimales.

\section{Plataformas para las parábolas}

En la torre se disponen dos tipos de parábolas, las destinadas a enlaces fijos y las que sirven a enlaces moviles.

Para las segundas se construyen dos plataformas a las cotas $+156,60 y+152,60 \mathrm{~m}$.

Son losas anulares macizas con un vuelo a partir del fuste de $3,50 \mathrm{~m}$.

Están previstas para una sobrecarga móvil de $400 \mathrm{~kg} / \mathrm{m}^{2}$.

En su perimetro se dispone una barandilla de acero galvanizado.

La solución más adecuada por el escaso vuelo de las losas es la de conexión empotrada al fuste. Este se refuerza en la zona de empotramiento para absorber los momentos adicionales que no son de gran magnitud. Las losas tienen un perfil transversal acartelado con un espesor minimo en el extremo del voladizo de $15 \mathrm{~cm}$ y máximo en la zona de contacto con el fuste de $45 \mathrm{~cm}$.

La armadura principal de la losa es Iógicamente la ra-

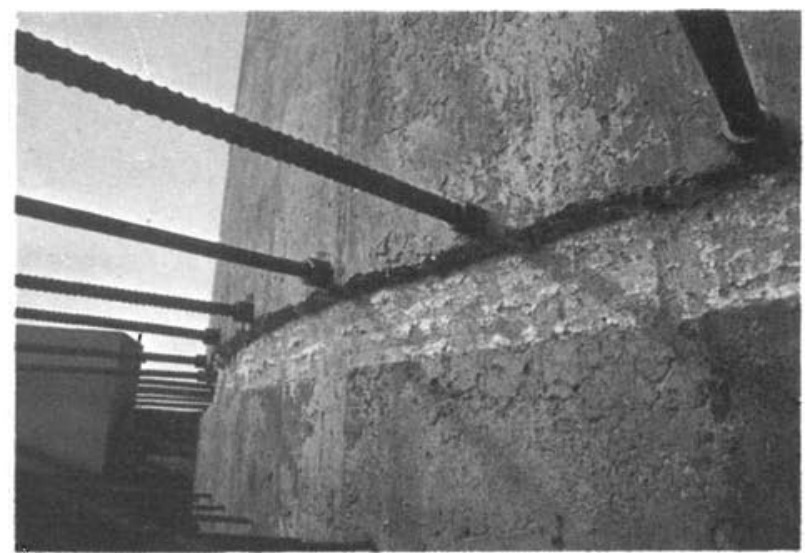

dial y su determinación se ha realizado conforme a la "Instrucción EH-80", empleándose un hormigón $\mathrm{H}-250$ y acero $\mathrm{AE}-42$.

Con el fin de no interrumpir el deslizamiento del fuste, la conexión de las losas al fuste se ha realizado mediante anclajes especiales. Durante el deslizamiento se dejaron en la pared del fuste, a las cotas correspondientes a las plataformas, 88 anclajes roscados para recibir barras tipo Dywidag de $\varnothing 25$ en cada una de las plataformas para parábolas móviles.

Posteriormente al ejecutar las losas se roscaban las barras a los manguitos con las correspondientes tuercas de seguridad, y esta armadura, solapada con la de la losa, constituye la armadura de empotramiento de las mismas.

Para las parábolas fijas se han previsto también dos plataformas situadas a las cotas $+146,60$ y $+140,60 \mathrm{~m}$ con $4,5 \mathrm{~m}$ de vuelo la primera y $5 \mathrm{~m}$ la segunda.

Estas plataformas son también losas anulares macizas. Soportan una sobrecarga móvil de $600 \mathrm{~kg} / \mathrm{m}^{2}$.

En estas losas se han dejado 10 orificios rectangulares para el paso de los cables de alimentación de las parábolas.

Aunque el vuelo es mayor que el de las plataformas para enlaces móviles se ha adoptado la misma solución estructural, mediante empotramiento de las losas en el fuste. En este caso los momentos radiales son de mayor consideración y se ha tenido la necesidad de aumentar el espesor de la pared del fuste para con el necesario refuerzo de armaduras absorber los momentos de empotramiento.

Estas losas tienen un perfil transversal acartelado con un espesor minimo en el extremo del voladizo de $17 \mathrm{~cm}$ y máximo en la zona de contacto con el fuste de $60 \mathrm{~cm}$.

El método de dimensionado y cálculo asi como la conexión de armaduras del fuste es idéntico al de las plataformas de parábolas de enlaces móviles.

El número de anclajes roscados para recibir barras de tipo Dywidag ha sido de 98 para la losa de $4,5 \mathrm{~m}$ de vuelo y de 125 para la de $5 \mathrm{~m}$ de vuelo.

Al ser las parábolas un elemento de enlace con otras situadas a decenas de kilometros, se limitaron los án- 


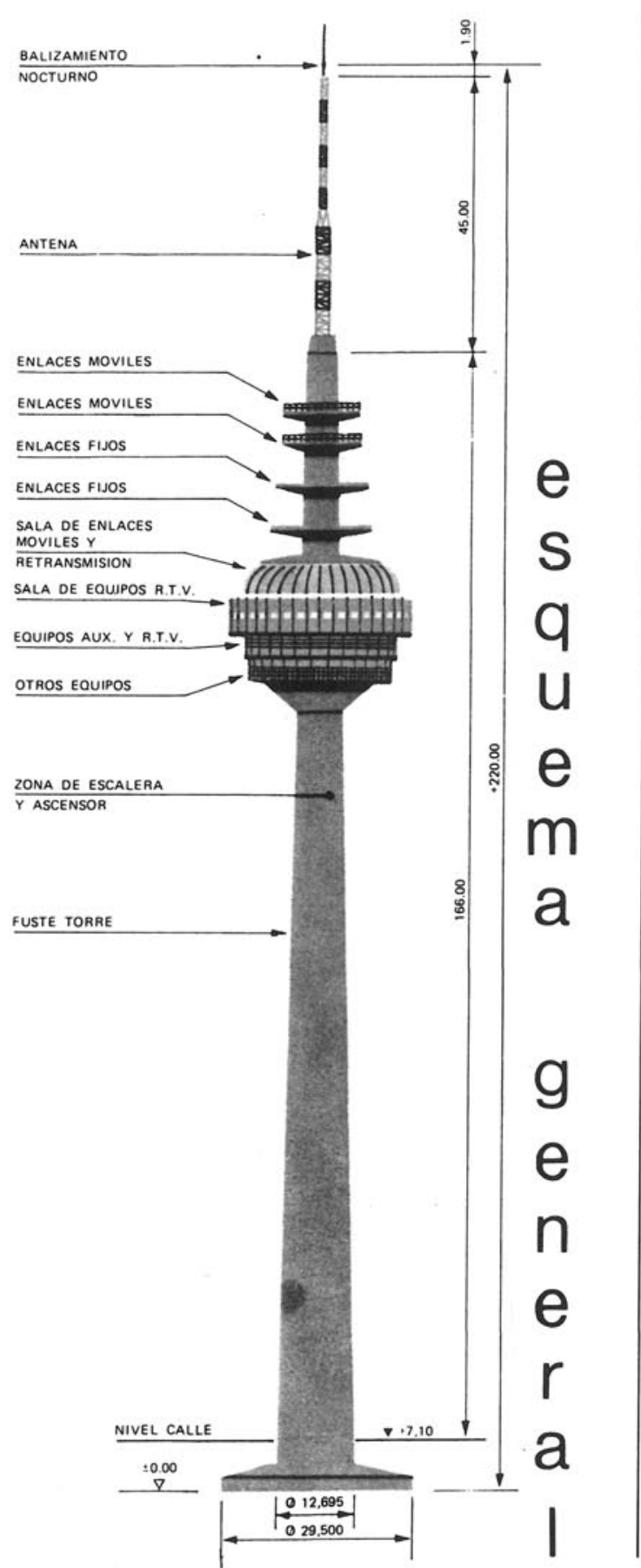

- A las cotas $+136,00,+141,00,+147,00,+153,00$ $y+157,00$ cinco aberturas de $2,55 \mathrm{~m} \times 1,10 \mathrm{~m}$ de ancho para acceso a las cuatro plataformas de parábolas y a la mansarda.

Para paso de cables del interior del fuste a plataformas y viceversa se han dejado orificios en todas las plantas, cuatro por planta y distintas dimensiones.

El fuste de hormigón se ha dimensionado siguiendo la teoria de tensiones admisibles que preconiza la norma americana $\mathrm{ACl}-307-79$.

Las acciones consideradas han sido:

- Peso propio y carga permanente del fuste.
- Peso propio y sobrecargas de la antena metálica sobre coronación del fuste.

- Peso propio, cargas permanentes y sobrecargas de todas las plataformas tanto abiertas como cerradas.

- Parte del peso propio, cargas permanentes y sobrecargas del núcleo de ascensor y escaleras, que no va directamente a la cimentación.

- Carga de nieve de $80 \mathrm{~kg} / \mathrm{m}^{2}$ en todas las plataformas abiertas y en la cubierta de las cerradas.

- Acción del viento con una presión dinámica en toda la altura de la torre de $150 \mathrm{~kg} / \mathrm{m}^{2}$. Considerándose un factor eólico para las parábolas de 2 y para las superficies cilindricas del fuste de 0,7.

El factor de esbeltez considerado para el fuste ha sido de 1,29 .

Se ha tenido en cuenta el efecto del viento sobre la sección transversal del fuste "ovalización" considerando la distribución horizontal del viento recomendada en las normas internacionales como del CICIND.

Se estudió la influencia de los torbellinos de Von Karman, en las oscilaciones de la torre, en el sentido transversal a la dirección del viento.

La acción sismica, a pesar de que Madrid es zona de baja intensidad sismica, se contemplo conforme a la Norma Sismorresistente PDS-1 Parte A-Normativa, contemplándose la superposición de los tres primeros modos fundamentales de oscilación. Además se calcularon los momentos sismicos por el método recomendado por la norma ACl-307-79 considerando como factor de zona $z=0,25$ y un factor de uso $U=2,00$.

Las hipótesis de cálculo han sido:

1) Hipótesis Normal:

Presión dinámica del viento de $150 \mathrm{~kg} / \mathrm{m}^{2}$. Valor caracteristico.

Cargas permanentes y sobrecargas de uso. Valor característico.

2) Hipótesis Extrema:

Presión dinámica del viento de $1,75 \times 150 \mathrm{~kg} / \mathrm{m}^{2}$. Valor característico.

Cargas permanentes y sobrecargas de uso.

Valor caracteristico.

3) Hipótesis Sismica:

Acción sismica. Valor característico.

Cargas permanentes y sobrecargas de uso. Valor caracteristico.

4) Hipótesis de Construcción:

Presión dinámica del viento de $150 \mathrm{kp} / \mathrm{cm}^{2}$. Valor caracteristico.

Cargas permanentes. Valor caracteristico.

En cada una de estas hipotesis se ha determinado para cada sección considerada (un total de veintidós secciones) la tensión en el hormigón y la tensión en el acero, limitándose dichos valores a los dados como admisibles por la norma $\mathrm{ACl}-307-79$ para un hormigón de una resistencia especificada de $320 \mathrm{~kg} / \mathrm{cm}^{2}$ y un acero de limite elástico $4.200 \mathrm{~kg} / \mathrm{cm}^{2}$ y que son: 
1) Hipótesis Normal:

Tensión admisible en el hormigón: Tensión admisible en el acero:

$80 \mathrm{kp} / \mathrm{cm}^{2}$ $1060 \mathrm{kp} / \mathrm{cm}^{2}$.

2) Hipotesis Extrema:

Tensión admisible en el hormigón: Tensión admisible en el acero:

3) Hipótesis Sismica:

Tensión admisible en el hormigon: Tensión-admisible en el acero:

$120 \mathrm{kp} / \mathrm{cm}^{2}$. $1270 \mathrm{kp} / \mathrm{cm}^{2}$.

4) Hipótesis de Construcción:

Tensión admisible en el hormigón: Tensión admisible en el acero:

$96 \mathrm{kp} / \mathrm{cm}^{2}$ $1800 \mathrm{kp} / \mathrm{cm}^{2}$.

Para la determinación de las máximas deformaciones del fuste se han tenido en cuenta el efecto de inclinación de la cimentación con un valor máximo calculado de 0,031 grados sexagesimales, y el giro producido por efecto de soleamiento estimado en 0,25 grados sexagesimales para una diferencia de temperaturas entre las caras expuestas al sol y la opuesta de $25^{\circ} \mathrm{C}$.

En las zonas de aberturas y de conexion de plataformas y placas de anclajes se ha estudiado el efecto local que dichas perturbaciones producian, introduciendo los oportunos refuerzos locales de armaduras en el fuste.

\section{Cimentación}

Siguiendo las recomendaciones del estudio geotécnico, se decidió por la solución de losa circular maciza.

La parte inferior de esta losa tiene un diámetro de 29,50 metros con un canto de 2 metros. La parte superior es un tronco de cono de $16,5 \mathrm{~m}$ de diámetro en su cota más alta y una altura de $1,50 \mathrm{~m}$.

En el centro de la losa se efectuó un vaciado de $2 \times 2 \mathrm{~m}$ y $1 \mathrm{~m}$ de profundidad como foso del ascensor.

La losa asienta sobre una capa de regularización de hormigón $\mathrm{H}-150$ de $15 \mathrm{~cm}$ de espesor.

El encofrado perdido se efectuo con pared circular de bloques de hormigón.

La zona de contacto terreno-losa se inyecto posteriormente con lechada de cemento para evitar filtraciones de agua superficiales a capas más profundas donde se detectaron unas arcillas ligeramente expansivas.

La cimentación se ha calculado en cuanto a sus dimensiones de forma que no aparezcan zonas inactivas en ninguna de las hipotesis consideradas.

La máxima presión que se transmite al terreno en la hipótesis normal es de $3,80 \mathrm{kp} / \mathrm{cm}^{2}$. También en esta hipotesis el coeficiente de seguridad al vuelco es de 11,3 .

El hormigón de la losa de cimentación es $\mathrm{H}-250$ y la armadura $\mathrm{AE}-42$.

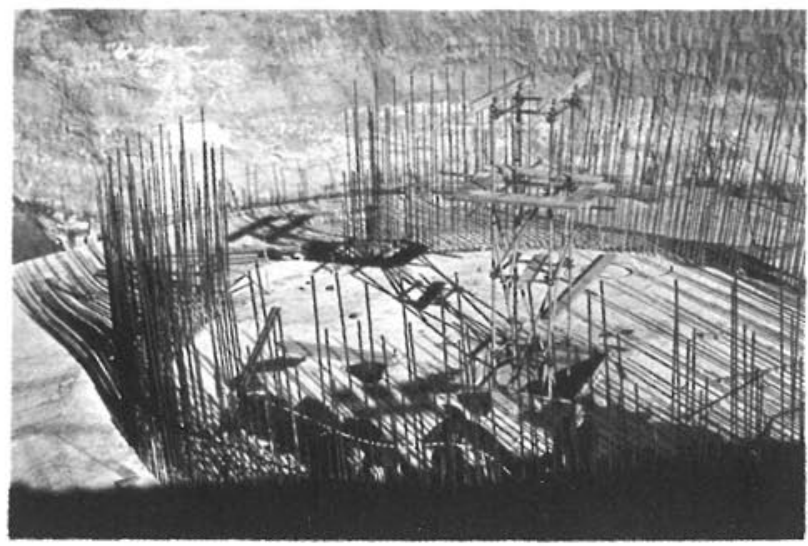

El módulo de deformación del terreno es de $E s=1.000$ $\mathrm{kp} / \mathrm{cm}^{2}$.

El cálculo de armaduras se ha realizado según la teoria de G. Markus.

\section{ACABADOS E INSTALACIONES}

\section{Antena}

Como ya se ha comentado es una estructura metálica galvanizada con pintura blanca y roja para balizamiento.

Dispone en su interior de una escalera de patas con aros protectores para acceso a la zona superior de la antena. Adosados a dicha escalera se disponen los soportes para los cables que alimentan los elementos emisores propiamente dichos.

\section{Plataformas para parábolas}

Su acabado se ha realizado en hormigon visto.

En las dos plataformas superiores se ha instalado una barandilla metálica galvanizada y bancadas metálicas para soporte de parábolas.

Las dos plataformas inferiores llevan un tubo de seguridad adosado al fuste y dos carriles metálicos donde anclar las parábolas. Todos estos elementos galvanizados.

\section{Cesta}

En ella se distinguen cuatro niveles:

Nivel 1._Planta destinada a "otros servicios".

Su suelo a la cota $+118,40$ es a la vez la losa de cierre de la copa, de $20 \mathrm{~cm}$ de espesor, y sobre él se coloca una capa de suelo industrial de hormigón con cuarzo. El cerramiento de esta planta se ha realizado a base de paneles curvos prefabricados de GRC (glass reinforced concrete).

Desde esta planta se accede a una pasarelá exterior, de perfiles metálicos galvanizados y pintados, prevista para una sobrecarga de $1.000 \mathrm{~kg} / \mathrm{cm}^{2}$. 


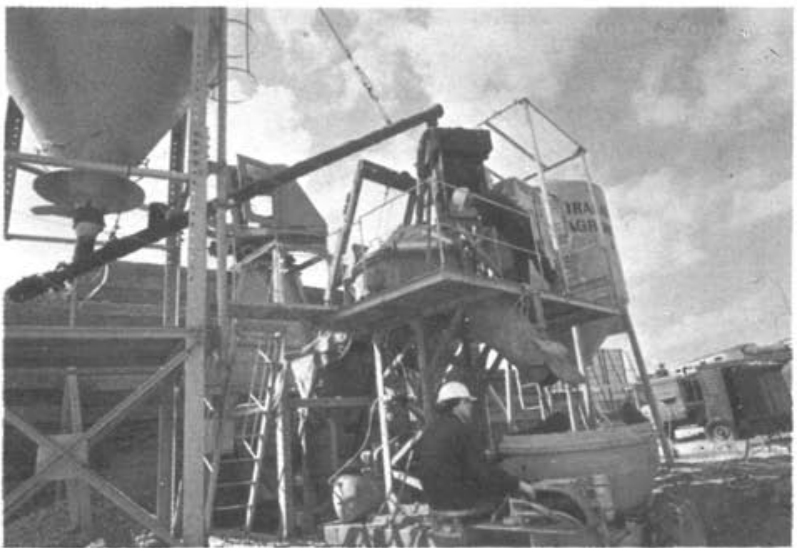

clos. Dicha planta, semiautomática, posee una mezcladora activadora de eje vertical, que fabrica un hormigón muy homogéneo, de gran plasticidad y calidad, debido al flujo producido por sus palas especiales.

Dadas las exigencias en cuanto a resistencia $(320$ kilogramos $/ \mathrm{cm}^{2}$ Normas $\mathrm{ACl}$ ) y al aspecto del hormigón visto (obra muy visible y espectacular); la dosificación fue de $400 \mathrm{~kg} / \mathrm{m}^{3}$ de cemento P- 450 , con árido rodado del Jarama de tamaños $5-20 \mathrm{~mm}$ y 20-35 mm, y arena de rio.

La planta estaba situada a unos $20 \mathrm{~m}$ de la base del fuste.

No se utilizó ningún aditivo en el hormigón. Solamente un producto de curado en superficie.

El transporte se hacia mediante volquete hidráulico hasta el cazo de hormigón, en la base de la torre. La puesta en el molde del hormigón se hacia en un tiempo máximo (coronación) de 4 a 5 minutos. Los asientos en cono de Abrams eran de 6-9 cm a pie de amasadora, y 4-6 al colocarlos en el molde.

Para la elevación de personas y material se dispusieron dos cabrestantes con carga por el interior del fuste y una grúa de tipo derrick para izado del acero en redondos por el exterior.

Los cabrestantes estaban situados en unas casetas preparadas a tal efecto a unos $15 \mathrm{~m}$ desde la base de la torre, perfectamente protegidas de la caida de materiales.

El cabrestante para personas, de $2.500 \mathrm{~kg}$ de capacidad de elevación con dos velocidades, la mayor de $90 \mathrm{~m} / \mathrm{min}$ tenia $80 \mathrm{CV}$ de potencia y estaba provisto de un cable $\varnothing 19 \mathrm{~mm}$ antigiratorio. Dicho cabrestante se ha equipado con un freno de emergencia de $80 \mathrm{~m} / \mathrm{kg}$ de par de frenada, ralentizador por corrientes de Foucault y dispositivo de hombre muerto. La jaula de personal que iza, va provista de dos cables guias, con un sistema de trenzado continuo y con un paracaídas de mordazas. Igualmente se disponia de finales de carrera electromagnéticos y células fotoeléctricas para la regulación de la subida o bajada.
El cabrestante para hormigon y otras cargas tiene $2.000 \mathrm{~kg}$ de capacidad, con cables de $\varnothing 13 \mathrm{mili}$ metros y una potencia total de $107 \mathrm{CV}$; la velocidad máxima de las tres que posee es de 88 metros $/ \mathrm{mi}$ nuto.

El fuste se comenzo a deslizar el dia 7 de abril y se terminó el 2 de junio de 1981, con una duración total de 56 dias para $170,43 \mathrm{~m}$ de altura a partir de la losa de cimentación, incluyendo dos paradas: la primera a los $15 \mathrm{~m}$ de altura para el cambio del sistema de hormigonado, con bombas hasta esta altura, al sistema definitivo de cabrestantes y la segunda, para permitir la formación del retranqueo en el fuste en la cota $+113,40 \mathrm{~m}$. La duración real del deslizamiento fue de 44 dias, con lo que la elevación media diaria real fue de $3,87 \mathrm{~m}$.

Entre los equipos auxiliares y de protección pueden destacarse:

- Sistema de televisión en circuito cerrado con una cámara en la plataforma de trabajo y dos monitores en los puestos de cabrestantes, para el control de las operaciones de izado.

- Sistema de comunicación cabrestantes-encofrado a base de genefonos (dos lineas independientes).

- Sistema de compuertas hidráulicas para entrada de jaula de personas y materiales, incluyendo el compresor.

- Pararrayos ionizante provisional.

- Balizamiento provisional e iluminación de los tres niveles de trabajo del encofrado deslizante.

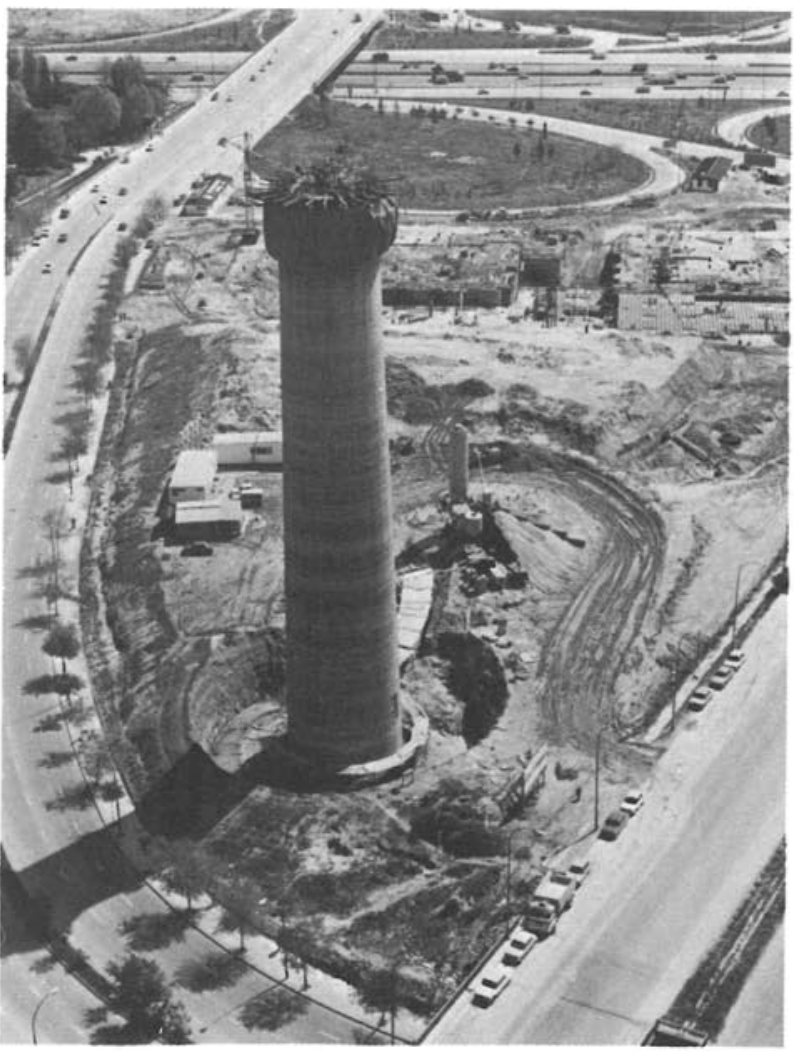


- Protección perimetral de lonas ignifugas en todo el encofrado deslizante.

- Sistema de extinción de incendios con extintores de $\mathrm{CO}_{2}$.

Durante el deslizamiento se dejaron situados en el fuste los huecos para puertas en plataformas, balizamiento y ventanas para entrada de cables; anclajes para la estructura metálica de plataformas cerradas en el encofrado de la copa; anclajes especiales tipo Gewi para el acero en redondos de la armadura de plataformas abiertas, asi como anclajes para posibles antenas intermedias.

\section{c) Construcción de la copa}

Dado lo ajustado del presupuesto de la obra se habia desechado la posible utilización de una grúa torre de pluma abatible tipo HBK o similar adosada al fuste por el exterior. Se estudio y decidio el montaje de una grúa Potain P-428 ligeramente modificada para este trabajo, pero colocada en la parte superior del fuste por el interior y anclada a dos plataformas en los últimos metros del fuste.

Para ello y mediante un andamiaje auxiliar en la coronación del fuste, de $15 \mathrm{~m}$ de altura y la utilización del cabrestante, se izó la grúa en módulos, según una compleja pero rápida secuencia, hasta su completa instalación.

Se habian estudiado todas las cargas que habian de ser izadas con esta grúa hasta la terminación de la

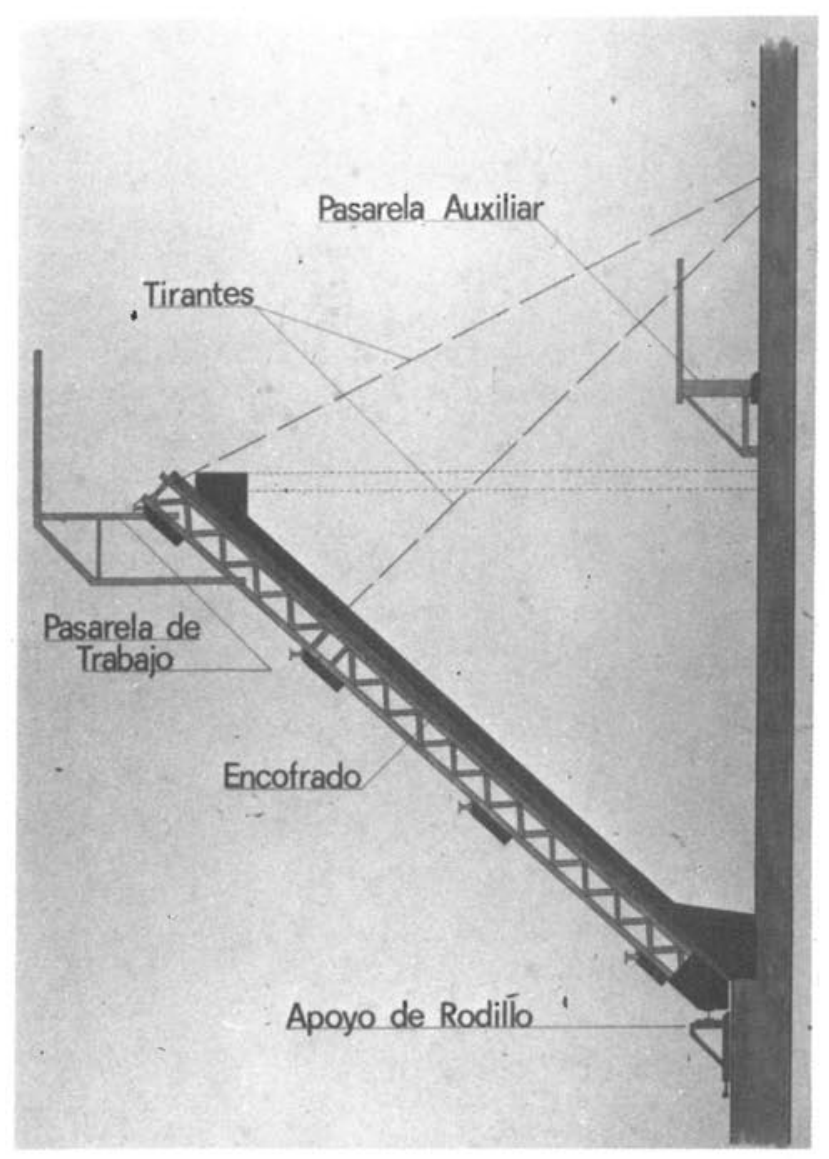

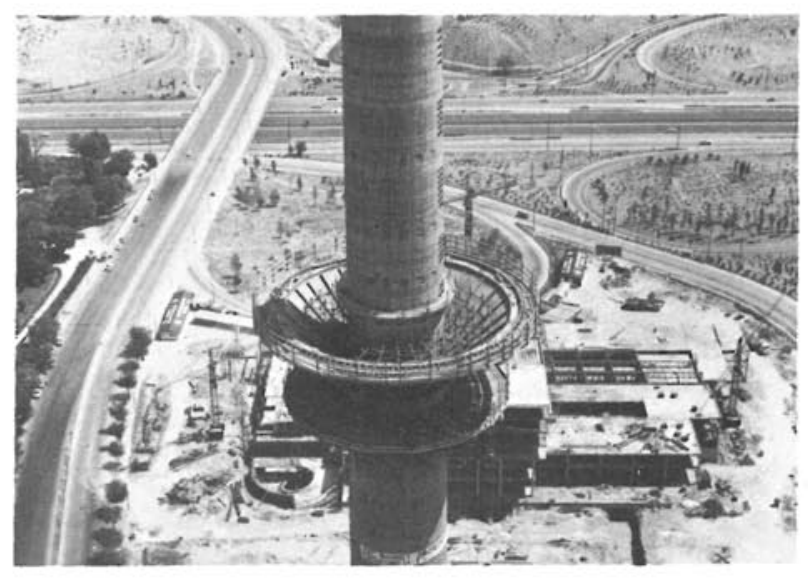

obra, de forma que con su capacidad de $2.000 \mathrm{~kg}$ en punta, con radio de $18 \mathrm{~m}$ y un giro de $360^{\circ}$, era suficiente.

El peso total del encofrado de la copa eran 20.000 kilogramos aproximadamente.

Paralelamente al desmontaje del encofrado deslizante, se procedia al montaje previo del encofrado de la copa, en el suelo, asi como de la plataforma auxiliar.

Para el encofrado de la copa, en forma de tronco de cono invertido, se utilizo un sistema de vigas resistentes de madera en celosia tipo PT. 70V dispuestas sobre cuatro correas circulares articuladas de acero, formando en total 14 sectores. Sobre las vigas en celosia se entablo, y posteriormente se coloco, la piel del encofrado a base de tablero fenólico.

El montaje en el suelo de este encofrado se preparó sobre andamiaje tubular.

Para poder situar en su cota el encofrado de la copa se preparó igualmente en el suelo una plataforma horizontal circular de trabajo, dividida en 16 sectores, que se izaron con la grúa y anclaron en los dispositivos dejados en el fuste. Desde esta plataforma se colocaron los apoyos de rodillo para la correa articulada inferior del encofrado de la copa. Se izaron los módulos de dicho encofrado y se fueron apoyando a la vez que se colocaban los tirantes contra el vuelco y los perfiles contraviento por cada módulo. Una vez terminada esta operación, se colocó la armadura de la copa y se procedió a hormigonar.

Para la losa de cierre de la copa, suelo de la primera plataforma cerrada a la cota $+118,40$, se utilizó un andamio auxiliar apoyado en el interior de la copa, sobre el que se preparó el encofrado de la losa, colocándose posteriormente la armadura y el hormigon.

d) Montaje de la estructura de escalera y núcleo de ascensor

Mientras se procedia a la construcción de la copa y de las plataformas cerradas, en el interior del fuste se montaba la escalera y el núcleo de ascensor con 
móviles en los planos radiales, definidos por cada brazo y el eje vertical de la construcción, realizan con sus movimientos las tres funciones siguientes:

1. a Adaptarse a la inclinación de los paramentos mediante accionamiento mecánico, con lo que se consiguen las pendientes de los paramentos con respecto al eje vertical del encofrado y cuyos valores fueron los siguientes:

De cota $-4,43$ a cota $+97,5$ pent. $2,50 \%$

De cota $+97,50$ a cota $+113,4$ pent. $0,00 \%$

De cota $+113,4$ a cota $+166,0$ pent. $1,25 \%$

2. ${ }^{\text {a }}$ Adaptarse al espesor de pared, operación que se realiza mecánicamente con desplazamientos máximos relativos entre encofrados interior y exterior de $2 \mathrm{~mm}$.

Habiéndose obtenido reducciones del espesor de muro de $208 \mathrm{~mm}$ desde la cota $-4,43$ a la cota $+97,50$, aumentos de $168 \mathrm{~mm}$ entre las cotas $+97,5$ y $+113,40$, en esta cota se hizo una reducción brusca de $230 \mathrm{~mm}$ para apoyo anular de la copa de hormigón, quedando el espesor de pared en $360 \mathrm{~mm}$.

Desde la cota $+113,40$ hasta la cota $+146,60$ se mantuvo un espesor constante de $360 \mathrm{~mm}$ y desde esta cota hasta la cota $+152,60$ el espesor de pared se redujo en $60 \mathrm{~mm}$ permaneciendo constante hasta la cota final $(166,00$ metros).

3. ${ }^{\text {a }}$ Adaptarse al diámetro en cada cota de forma continua mediante accionamiento hidráulico simultáneo en cada punto del contorno, por desplazamiento radial, como anteriormente se dice, de los pórticos sustentadores del encofrado propiamente dicho, habiendo comenzado con un diámetro de $12,736 \mathrm{~mm}$ para coronar con un diámetro de $5,865 \mathrm{~mm}$.

b) Encofrado propiamente dicho..-Formado por chapas metálicas rectangulares que permiten la consecución de paramentos completamente circulares. De la calidad de estas chapas depende la continuidad en la adaptación del molde a los paramentos; han de ser, por consiguiente, muy elásticas y resistentes a las deformaciones permanentes.

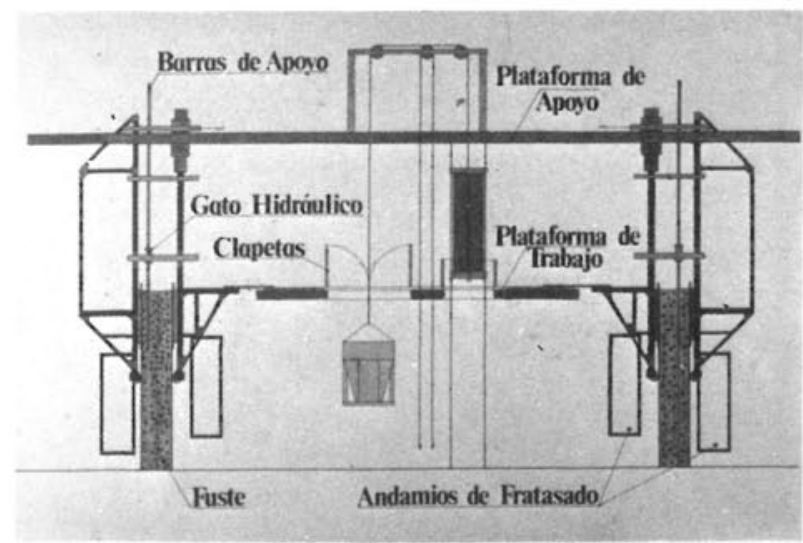

Estas chapas se adaptan a tres tubos, también elásticos, cuya deformación es forzada por los porticos al desplazarse radialmente por los brazos de la estructura portante.

c) Plataformas principales de trabajo interior y exterior.-Situadas a la altura del borde superior del encofrado, metálicas y variables en diámetro. Desde ellas se realizan las operaciones de recepción -a través de compuertas hidráulicas-, del hormigón, colocación del mismo, vibrado, colocación de armaduras y otros elementos de anclaje, pasamanos, señalización, etc. asi como todas las operaciones de manejo del encofrado deslizante.

d) Plataformas secundarias interior y exterior. -Situadas a $1,8 \mathrm{~m}$ por debajo del borde inferior del encofrado, en ellas se realizan las operaciones de acabado de paramentos, curado de los mismos, y vigilancia del hormigón a la salida del encofrado.

e) Equipo de elevación. -EI encofrado se elevó mediante 8 gatos oleohidráulicos de 6 t y 16 de 3 t, que trepan por varillas calibradas de 32 y $25 \mathrm{~mm} \varnothing$ respectivamente. Para la reducción del diámetro se emplearon 24 gatos de $3 \mathrm{t}$. Estos gatos fueron accionados mediante bomba eléctrica a través de mangueras flexibles de alta presión.

Los intervalos de acción de la bomba, previamente fijados, se cumplen automáticamente mediante un temporizador o emisor de impulsos.

El encofrado queda detenido automáticamente en aquellas cotas en que haya que realizar operaciones de reducción de diámetro, reducción del espesor de pared, etc.

\section{Verticalidad}

El control de verticalidad se hizo mediante plomada cenital, de optica pendular de gran precisión; las mediciones se obtienen con un error máximo de 土 $1 \mathrm{~mm}$ en 150 metros.

El encofrado va provisto de una referencia central de verticalidad y dos en los extremos de un diámetro que acusan cualquier giro del encofrado alrededor del eje de simetria vertical de la torre. De esta forma se pone de manifiesto con absoluta precision cualquier desviación del encofrado, lo que permite su inmediata corrección.

El intervalo de comprobación o corrección dependerá, en cada caso, de diversos factores tales como condiciones climatológicas, velocidad de deslizamiento e incluso dilataciones térmicas producidas por un fuerte soleamiento de una zona de la pared. Cualquiera de ellas puede hacer aconsejable una reducción en los intervalos de control.

Las correcciones nocturnas se realizan por el mismo procedimiento mediante la iluminación adecuada de las referencias de verticalidad o giro.

Al comienzo del deslizado las citadas referencias se fijan en la cimentación donde se colocan los puntos de estación fijos que ya no se abandonarán durante todo el deslizamiento. 


\section{MEDIDAS PREVENTIVAS DURANTE SU CONSTRUCCION}

\section{Iniroducción}

La construcción de la Torre de RTVE en Madrid (primera obra de este tipo realizada en España), en función a sus caracteristicas y emplazamiento, entraña además de los riesgos generales de toda construcción, unos riesgos especificos para trabajadores y terceros.

Su altura y yoladizos implican riesgo de caida de personas, materiales y objetos, 10 cual unido a la velocidad del viento, y a la proximidad a dos avenidas importantes y su confluencia, han hecho que hubiese que extremar las precauciones al máximo.

La filosofia preventiva en esta obra ha seguido las lineas de la Seguridad Integrada. La Jefatura de Obra y el Staff de Seguridad han coordinado sus actuaciones adelantándose a cada fase de ejecución de forma que los sistemas de producción llevasen incorporadas las medidas de seguridad idoneas; bien entendido que factores tales como variaciones de proyecto, definiciones casi inmediatas a la ejecución, plazos, etc., han hecho que no siempre se pudiese seguir dicha filosofia como seria de desear.

A grandes rasgos, las actuaciones preventivas han sido las siguientes:

\section{Prevención General}

El elemento humano ha jugado un papel de especial importancia teniendo el personal mucha experiencia en obras de gran altura, y disponiéndose de los especialistas adecuados a cada caso.

La actuación técnica ha seguido el Plan de Seguridad para deslizantes de gran altura, y ha integrado la seguridad en el plan de cada fase de ejecución (copa, plataformas, escaleras, grúa, etc.).

Las protecciones colectivas e individuales, la señalización y la lluminación, han sido las que requeria cada trabajo, y las revisiones y mantenimiento de instalaciones, electricidad, maquinaria, y material de seguridad, se han realizado con la periodicidad pertinente.

\section{Prevenciones especificas de cada fase de obra}

\section{a) Excavación y cimentación}

Taludes adecuados a la excavación de la zapata circular, acceso en rampa, señalización y acotación de bordes, y vigilancia continuada frente a terceros.

Para la colocación de la armadura de la zapata, se utllizaron las pasarelas adecuadas en cada caso.

El hormigonado de la zapata que fue continuo se realizo con la iluminacion adecuada en las horas nocturnas, y la situación de camiones y bombas de hormigonar se hizo de forma que no surriese el talud de la excavación.

b) Fuste

A continuación se montó el encofrado deslizante del fuste, con todos los sistemas de protección co- lectiva incorporados de forma que desde el comienzo del deslizamiento no hubiese más que vigilar la correcta disposición de los medios de protección.

El encofrado disponia de tres plataformas a distinto nivel: además de disponer cada una de las barandillas perimetrales, se colocaron lonas ignifugas que cubrian todo el exterior y embolsaban por su parte inferior la plataforma exterior de fratasado, de forma que no solo protegian de la caida de pequenos objetos y evitaban el posible vertigo sino que ayudaban en los momentos de reducir barandillas y plataformas en función a la reducción del diáme. tro del fuste.

La plataforma interior de fratasado se protegio, ade. más de con barandillas, con malla metálica.

Las escaleras de comunicación entre plataformas estaban completamente protegidas.

El acceso de personal desde la base a la plataforma principal del encofrado se hacia por medio de aparato elevador con los sistemas pertinentes de seguridad: freno paracaidas, finales de carrera, etc. Entre la zona de mandos y la plataforma se disponia de circuito cerrado de televisión, teléfono y radioteléfono. El cabrestante disponia de freno de emergencia y mando de hombre muerto.

Se disponia de cabrestante independiente para elevación de hormigón y pequeños materiales.

La ferralla se elevaba exteriormente por medio de un derrick situado en la plataforma superior.

Las clapetas de entrada en la plataforma de ambos elevadores funcionaban con accionamiento hidráulico, y al quedar abiertas tenian incorporadas barandillas protectoras.

Con un sistema de poleas y pesos se variaba auto máticamente la longitud de los cables-guia de la cabina (en función a la variación de la cota del encofrado) y se mantenia la tensión de los mismos.

Las plataformas disponian de extintores y de lluminación artificial dado que la construcción del fuste ha sido continua, a base de distintos turnos de trabajo.

A nivel del terreno se tomaron las siguientes medidas:

Vallado del solar de la obra.

En funcion a la posible, aunque no probabte, caida de objetos, y teniendo en cuenta la zona de influen. cia de la parábola descrita por los mismos, se tomaron las medidas que se exponen a continuación:

Las siguientes tres zonas de acceso y paso: desde la entrada de la obra a la zona de oficina, vestuario, comedor, servicios y almacén, desde la zona de mandos de cabrestantes y la planta de hormigonado al fuste, y la circunvalacion a la torre para acceder a la zona de carga de ferralla, se protegieron con pasillos de seguridad con su techo cubierto.

Las calles de O'Donnell y Sáinz de Baranda se dotaron de vallas y señalización (entre ellas luminosa) 
on las zonas de infuencia de posible caida de obletos, gestionando previamente los correspondiantes permisos municipales.

Asimismo, en la base de la korre se creo un lecho de seguridad y dos pasillos protegios superior y lateralmente, uno hasta acceso al elevador de personas, y orro hasta el de hormigon lque se ransportaba hasta el cazo con un dumper).

Dada la gran altura de la obra, está dotada a partir de la cota pertinente, de balizas para los aviones, y desde el comienzo del deslizamiento, de pararrayos; este ulimo demostro su eficacia el 28 de abril, dia en que hubo una fuerte tomenta en Madrid, ca. yendo un rayo sin comsecuenclas negativas.

\section{c) Grúa-torre para construcción de voladizos}

Terminado el deslizamiento del fuste de la torre, simultáneamente se desmontó el encofrado deslizante $y$ se crearon dos plataformas a distinto nivel para sujeción de una grua-tore en la parte superior, y otra platarorma en la cota máxima, sobrevolando el diametro del fuste, con objeio de disponer de espacio suficiente para realizar los trabajos en condiciones buenas y seguras.

Las plataformas donde va suleta la grua disponen de una estructura melálica de soporte, soldada a placas de anclaje previstas en el fuste; estas platam formas se montaron en condiciones de seguridad desde otra platabroma cuajada y colgada de elemenlos estructurales del encofrado.

Se estudio en su momento la posibilidad de colocar la grúa con helicoptero, pero dadas las dificulades técnicas y las no suricientes garantias de segurm dad se opto por elevar el mástl (en varios tramos) yerticalmente por el interior de la tore, unirlos pos. teriormente, a continuacion izar pluma y contrapluma de Sorma simllar, unir los elementos, elevar el mástll (con pluma y contrapluma colgadas) desde las platarormas citadas, desplegar pluma y contrapluma, y bajar posteriormente la grua hasta su cota de servicio.

Todo el estucio detallado del proceso de montaje llevaba integrado su plan de seguridad.

Asimismo se cambiaron los reenvios de la cabina de personas y el elevador interior de materiales, desde el encofrado hasta el interior de la platatorma superior; se crearon dos nuevas platatormas de desembarco de la cabina, lotalmente protegidas, una a nivel de la copa y otra a la cota $+135,60$ subiendo se hasta la cola + 166,00 por una escalera de pates con jaula protectora y descansillos reglamentarios.

d) Voladizos

Constan de la copa y un conjunto de plataformas cerradas en cuatro niveles, y de cuatro plataformas abiertas.

Los mayores riesgos, además de la gran altura, estriban, primero en la construccion de la copa que, al nacer del fuste, carecia de plataformas de raba10, y segundo en que las dos planlas sigulentes a la tapa de la copa sobrevuelan aspecto a la inme. diata inferior. d-1) Copa

Para la colocacion y desmontaje del encorrado de la copa de homigon, se creo una plataforma de rabalo por debajo del futuro encorrado. Esta platafor ma construidz y colocada en sectores que rodean la torre, se colgo y sujeto en anclajes especiales pre vistos en el lusle, de forma que la gria pudiese colocar los sectores directamente; no obstante, colocado el primer sector y accediendo desde una de las puertas del tusie, por medio de escalera, pasa. relas. y elementos salyacaidas unidos al cinurón de seguridad, la colocación de los sigulentes seciores lue más sencllia. Todos los sectores llevaban incorporada su barandilla descle el montale a nivel del terreno, de foma qua la proteccion de la plata roma quedase automáticamente montada.

La mitad de los sectores uno si y otro mo) van an clacos a fuste y disponen de una plataforma interior protegida para fijar correctamente los anclaies; la otra milad, alrernalivamente va sobre los seciom res cllados an primer lugar.

Posteriomente, y de forma parecida, se coloco el encorrado de la copa con platarorma permetral barandllas incorporadas.

Para la sujecion de los tensores del encorrado se coloco una pequena plataforma también por secto res y con barandilla incorporada.

El acceso desce el aparato clevador se hacía por una puerta dejada en el fuste, y con escalera inte. rior se subia a la platatoma de rabajo para cons. truir la copa. Posteriormente se quito el encotrado con la ayuda de la plataforma inferior y se construyo la lapa, probegiendo previamente con barandilla el perimetro.

La copa dispone de pequeños orillolos para paso de cables y desmontaje de encohrado y plataroma inte. rior, y de un hueco que posteriomente se cetrara, para retorno de los trabajadores que ayuden a des. montar el iltimo elemento de platatoma interior.

Simultáneamente se lba montando le scalera defi nitiva interior a la tore, tambien con esiudio de se. guridad integrado en el de montale.

\section{D-2) Platatormas cerradas}

Construida la lapa do la copa, se disponia ya de un piso definitivo on voladizo; no obstante, habia que montar las estructuras metálicas de las dos siguien. tes platatomas que sobrevuelan respecto a la copa, y los correspondientes forjados.

En un principio se estudio y proyecto un solisicado sistema especifico de redes y soportes de forma que su montaje y movimientos sucesivos no entra. ñasen riesgos.

No obstante, dadas entre otras las condiciones de plazo, espacio reducido, salida de los castilletes de apeo de las platatormas ce hornigon, atc. y dado lambien que las plataformas de estructura melálica dispondrian de encotrado perdido a base de chapa metálica ondulada, se opto, conjuntamente con la linea de produccion, por orro sistema de proteccion 


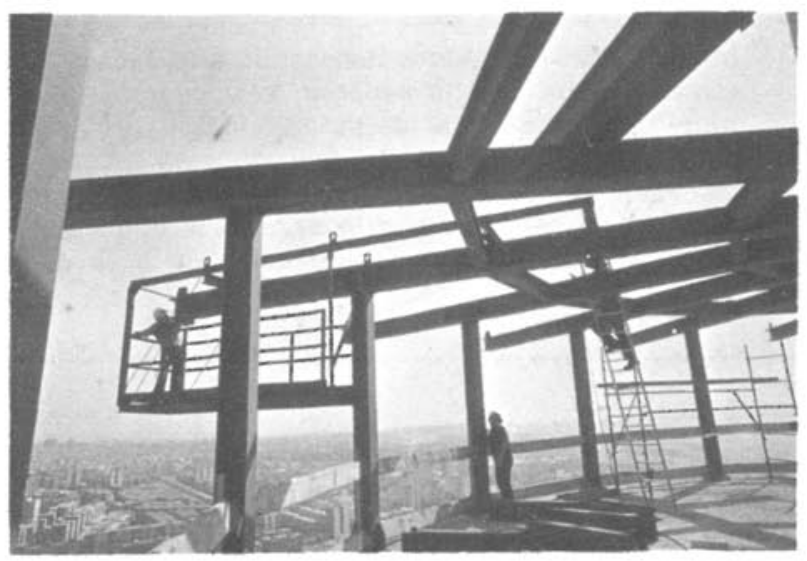

igualmente seguro y más adecuado a las caracteristicas de la obra.

Este sistema ha sido a base de plataformas de trabajo totalmente protegidas, y colgadas de las vigas radiales; montados los pilares y soldadas dichas vigas al pilar y a las placas del fuste, dos plataforformas (jaulas de longitud mucho mayor a su anchura y altura) se colgaban con la grúa a lo largo de dos vigas radiales contiguas. El acceso se hacia desde el forjado, y en ellas se trabajaba con toda seguridad en el montaje de las vigas perimetrales voladas.

Dichas plataformas llevaban dos barras verticales abatibles y una horizontal pasante, para su correcta sujeción a la viga que permitan posteriormente el desmontaje una vez colocadas las vigas perimetrales.

Entre los pilares, a su vez, se montaban barandillas.

Asimismo se ha dispuesto de otro tipo de jaulas protegidas que, soportadas entre dos forjados, dejaban la zona de trabajo en voladizo; estas jaulas han servido para colocar, entre otros, los soportes verticales del pasillo metálico que rodea a la copa, anclajes bajo vigas, etc.

\section{d-3) Plataformas abiertas}

Los apeos del encofrado disponian de barandilla en cada sector.

No obstante, en las dos primeras plataformas se colocó barandilla provisional (pues no disponian de definitiva) y en las dos últimas se montó lo antes posible su barandilla definitiva.

\section{e) Escalera definitiva}

Como se ha citado, se empezó a montar una vez instalada la grúa, y el sistema de seguridad iba integrado en el de montaje. Entre los soportes verticales, que forman a su vez el hueco del ascensor, se ha dispuesto una plataforma que además de ayudar a los trabajos, evitaba la caida por el interior, pues se iba elevando a medida que se montaba la escalera. Cada tramo de escalera, cogido con grúa, se presentaba desde el tramo anterior, por lo que unido a la plataforma citada, los trabajos se realizaban (ayudados de cinturón de seguridad) en buenas condiciones.

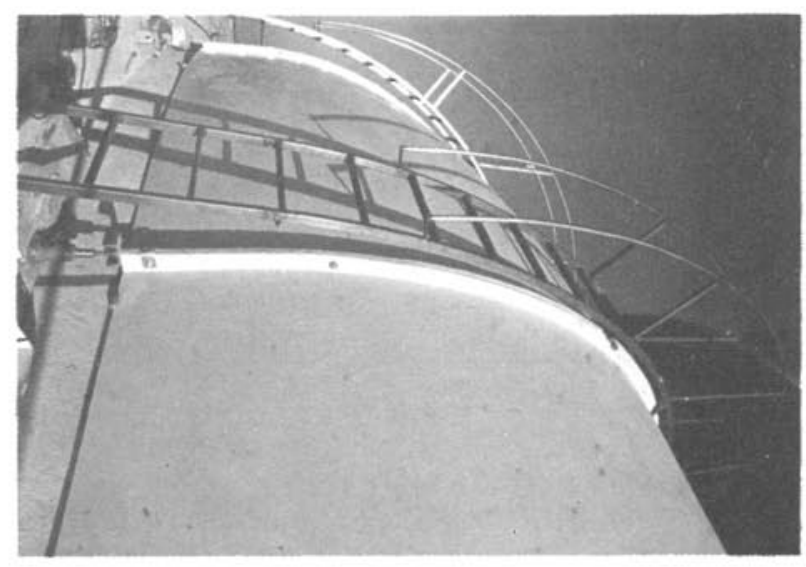

Como su construcción fue bastante rápida, el acceso desde el desembarco del elevador de personas a las plantas en voladizo, se ha realizado normalmente por dicha escalera; para ello se crearon accesos provisionales perfectamente protegidos a las distintas plantas (dado que los futuros desembarcos desde el ascensor definitivo a los distintos niveles, no se podian colocar todavia).

La escalera se montó hasta la cota que permite la grúa. A partir de dicha cota el acceso se realiza con escala vertical protegida y desembarcos igualmente seguros.

\section{f) Cerramientos}

La elevación de los elementos de cerramiento de la mansarda y la planta más saliente se ha realizado con la grúa-torre y disponiendo de los útiles necesarios.

En las plataformas retranqueadas la elevación se ha realizado con cabrestante situado en la planta a cerrar, ayudado por poleas de reenvio a nivel de techo de dicha planta.

El montaje del cerramiento tórico (que va del último nivel cerrado a la mansarda) se ha realizado desde dentro de la planta, ya que va desde un pretil interior a un perfil situado bajo la mansarda. No obstante, para las uniones de que dispone exteriormente, se ha construido una escalera con protección que se adapta a la forma del cerramiento.

El montaje de las piezas de cerramiento de la planta más saliente se ha realizado, interiormente, desde su forjado y el inmediato superior.

Los cerramientos de los dos niveles interiores también se han montado desde el interior de las plantas.

\section{g) Antena}

Su montaje se hizo por expertos en este tipo de trabajos. La elevación de los módulos de mástil se ha realizado con un plumin que al disponer de un sistema de trepado sobre la parte de antena construida, está siempre en la cota superior. 
Los montadores, por el interior de la antena, fueron ensamblando los elementos, y disponian de los medios de seguridad adecuados.

h) Galeria de unión Torre-Edificio de Informativos, $e$ Instalaciones

Las medidas de seguridad son las habituales en este tipo de obras, que no necesitan especial mención por ser conocidas.
No obsiante, reseñamos que la galeria se ha consiruido dando los taludes necesarios a la excavación, y señalizando la parte superior; el encuentro con ia torre ha implicado el desvio de la circulación de personas y camiones por el lado opuesto a la entra. da, así como la elevación de la base del aparato elevador de personas al que se accede actualmente desde el nivel del terreno (no desde la base de la torre).

\section{OBRA}

JOSE RAMON BURGOS MORCILLO

Ingeniero de Caminos. AGROMAN, Empresa Constructora.

ALFREDO CHAVES DE CASTRO

Ingeniero Técnico Industrial. YDE - AGROMAN

FELIX RODRIGUEZ HUIDOBRO

Ingeniero Técnico de Obras Públicas. AGROMAN, Em. presa Constructora, S. A.

\section{SEGUPIDAD}

FERNANDO MIRET CORRAL

Ingeniero Industrial. AGROMAN, Empresa Constructora, S. A.

\section{PROYECTO}

JOSE DEL SOLAR BERMEJO

Ingeniero de Caminos. KARMAN, Técnicas Especiales, S. A.

FERNANDO HUE GARCIA

Ingeniero de Caminos. DRAGADOS Y CONSTRUCCIO. NES, S. A.

\section{última publicación del IE.T.T.c.}

\section{bases para el diseño solar pasive}

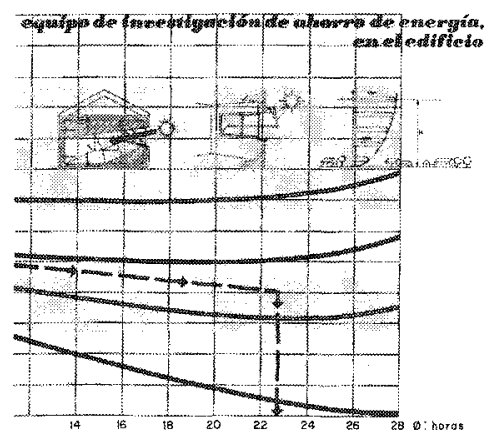

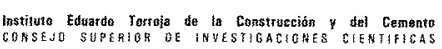

Equipo de Ahorro de Energía en el edificio

Dirección y coordinación: Arturo Garcia Arroyo

M. ${ }^{a}$ José Escorihuela José Luis Esteban José Miguel Frutos Manuel Olaya

Bernardo Torroja
Las dificultades de suministro y ef alto coste de los productos energéticos convencionales han despertado la atención de los usuarios, técnicos e industriales de la edificación hacia los procedimientos y sistemas en que se basa el aprovechamiento de otras fuentes alternativas de energía, principalmente la solar. Esto utores de este libro, arrastran los siguientes defectos: un mimético tecnologismo respecto de los sistemas convencionales que violenta las pe culiaridades de la energia solar (baja densidad y variabilidad en el tiempol, y una escasa selectividad en la aplicación de los sistemas y procedimientos pasivos dando origen a un ecumenismo arquitectónico solar, al margen de las condiciones climáticas y funcionales específicas de cada caso y lugar.

En este libro, utilizando criterios y metodología pedagógicos, se dan los fundamentos e instrumentos teórico-prácticos necesarios para el planteamiento de todo proyecto arquitectónico solar pasivo, de acuerdo con los principios éticos y económicos de conservación y ahorro de energía. Es decir: respeto de los presuptuestos bíoclimáticos, búsqueda de la máxima captación y acumulación de la radiación solar, $y$ esmero en el aislamiento térmico de los cerramientos.

Un volumen encuadernado en cartulina ibiza plastificada, a cinco colores, de $16 \times 23 \mathrm{~cm}$, compuesto de 216 páginas, 217 figuras, 87 gráficos, 19 tablas y 10 cuadros,

Madrid, 1983. Precios: España 2,100 ptas.; 30 \$ USA. 